\title{
Implementación de Sistema de Gestión de Calidad y Seguimiento al desempeño docente en un Instituto peruano
}

\author{
Implementation of a Quality Management System and Monitoring of teaching \\ performance in a Peruvian institute
}

Implementação de Sistema de Gestão da Qualidade e monitoramento do desempenho docente em Instituto peruano

\author{
Regina T. Martínez García \\ regina.martinezgarcia11@gmail.com \\ https://orcid.org/0000-0002-8693-8459
}

Universidad Nacional Mayor de San Marcos, Lima-Perú

\section{RESUMEN}

El presente artículo analiza la implementación de como un Sistema de Gestión de Calidad (SGC) y su influencia en el seguimiento al desempeño docente en el Instituto de Educación Superior Tecnológico Privado Comunidad Teológica del Perú. Para ello, se describe el comportamiento de las variables de acuerdo con las actividades que realizan los docentes, personal administrativo y los estudiantes. La metodología empleada fue bajo un enfoque cuantitativo, con un análisis correlacional-causal, de corte transversal. Como resultado se obtuvo en la prueba de inferencia que el coeficiente de correlación es de 0.885 , por lo cual se infiere que existe una correlación positiva alta entre las variables estudiadas. Para finalizar se puede concluir que la Implementación de un SGC sí influye en el seguimiento al desempeño docente.

Palabras clave: Calidad de la Educación; Supervisión; Desempeño del profesor; Perú

\section{ABSTRACT}

This article analyzes the implementation of a Quality Management System (QMS) and its influence on the monitoring of teaching performance at the Instituto de Educación Superior Tecnológico Privado Comunidad Teológica del Perú. For this purpose, the behavior of the variables is described according to the activities performed by teachers, administrative staff and students. The methodology used was under a quantitative approach, with a cross-sectional correlational-causal analysis. As a result of the inference test, the correlation coefficient was 0.885 , which indicates that there is a high positive correlation between the variables studied. Finally, it can be concluded that the implementation of a QMS does influence the monitoring of teacher performance.

Key words: Education quality; Supervision; Teacher performance; Peru

\section{RESUMO}

Este artigo analisa a implementação de um Sistema de Gestão da Qualidade (SGQ) e sua influência no monitoramento do desempenho do ensino no Instituto de Educación Superior Tecnológico Privado Comunidad Teológica del Perú. Para este fim, o comportamento das variáveis é descrito de acordo com as atividades realizadas pelos professores, pessoal administrativo e estudantes. A metodologia utilizada foi uma abordagem quantitativa, com uma análise transversal de causas correlacionais. Como resultado do teste de inferência, o coeficiente de correlação foi de 0,885 , o que significa que há uma alta correlação positiva entre as variáveis estudadas. Finalmente, pode-se concluir que a implementação de um SGQ influência de fato o monitoramento do desempenho dos professores.

Palavras-chave: Qualidade Educacional; Supervisão; Desempenho do Professor; Peru 


\section{INTRODUCCIÓN}

En la actualidad hablar de una educación de calidad, implica que las mayorías de organizaciones educativas consideren como objetivo la satisfacción de sus estudiantes, cumpliendo una serie de condiciones, requisitosy/o estándares de calidad; por ello, se requiere que cada una de las organizaciones realicen una evaluación de sus procesos mediante el Sistema de Gestión de Calidad para poder gestionar de una manera más óptima sus recursos, y dirigir una política de calidad, que permite lograr los objetivos y garantizar una mejora continua en su contexto organizacional.

En América Latina se realizan grandes esfuerzos para brindar una educación de calidad; por ello, los gobiernos crean y apoyan diversos espacios públicos y privados para acompañarla con un adecuado control y orientación dentro de las organizaciones, a través de auditorías internas o externas que garantizan la mejora de la calidad educativa; es importante mencionar algunas de las entidades comprometidas con la calidad: Comisión Nacional de Acreditación (CNA-Chile. 2006), Comisión Nacional de Evaluación y Acreditación Universitaria (CONEAU-Argentina, 1995), Consejo para la Acreditación de la Educación superior (COPAES-México, 2000, en de la Garza, s.f.), entre otros; todas ellas abrazan un enfoque de calidad educativa y orientan a las organizaciones a detectar y mantener los estándares que pueden tener con un manejo de excelencia, en este proceso de evidenciar su implementación. Es importante indicar que estas entidades internacionales cuentan con un compromiso de calidad y forman redes para concientizar a las autoridades, docentes, estudiantes y padres de familia; es decir a la sociedad, a fin de darle un valor a la educación con calidad.
En Perú, el compromiso de brindar un servicio de calidad es cada vez más exigente; así como en los países vecinos, el Estado peruano impulsó la creación de espacios para velar por ella, desde el control que emite la Superintendencia Nacional de Educación Superior Universitaria (SUNEDU) quien otorga el licenciamiento a muchas instituciones con condiciones básicas de calidad; además, del Sistema Nacional de Evaluación, Acreditacióny Certificación de la Calidad Educativa (SINEACE) que promueve la certificación en personas y la acreditación de programas de estudios; otro organismo regulador es el Ministerio de Educación (MINEDU), quiénes actualmente, cumplen el rol de veladores de la gestión educativa en las instituciones de Educación Superior Universitaria, Institutos y de educación básica. Esta realidad invita a las instituciones educativas a cumplir los requerimientos básicos y brindar un servicio de calidad, y para ello deben implementar un Sistema de Gestión de Calidad que garantice el cumplimiento de estándares de calidad, con adecuados controles y seguimientos a los indicadores de cada proceso determinado en cada contexto educativo.

En el Instituto Superior Tecnológico Privado "Comunidad Teológica del Perú" se tiene previsto pasar de titulación técnica a licenciatura, por ello, la Dirección ha tomado la decisión de implementar un Sistema de Gestión de Calidad desde el enfoque de la normativa ISO 9001:2015, esto con la finalidad de involucrar a los agentes (Directivos, administrativos, docentes y los estudiantes) y comprender que un Sistema de Gestión de Calidad, garantiza la eficiencia de diversos procesos, en este caso, el seguimiento al desempeño docente que es parte importante del proceso de enseñanza y aprendizaje en la institución. 
Entre los diversos factores causales de la necesidad de implementar un Sistema de Gestión de Calidad, se ha detectado que el personal en conjunto, desconoce del estándar $\mathrm{N}^{\circ} 6$ denominado "Sistema de Gestión de Calidad" del Modelo de Acreditación para programas de Estudios de Institutos y Escuelas de Educación Superior, que entre sus requisitos indica la necesidad de implementar un Sistema de Gestión de Calidad en la que se pueda definir las políticas, los objetivos y los planes de la institución; así como los mecanismos que brindan confianza y manejo de los procesos para la mejora continua.

$\mathrm{Si}$ esto persiste, lamentablemente no se podrá controlar diversos procesos, y en este caso el Seguimiento al desempeño docente traerá deficiencias en el control del proceso de rendimiento académico de los futuros profesionales en Teología; es decir, que la organización mantendrá mecanismos obsoletos, estáticos y funcionales para el control de las entradas y salidas de cada proceso; siendo un grave porque generaría una formación incoherente con el perfil de egreso. Definitivamente, el estatus quo del proceso de Seguimiento al desempeño docente perjudicaría a los estudiantes.

Por ello, se sugiere analizar como la implementación de un Sistema de gestión de calidad, que permita la constante evaluación influye en las mejoras al proceso de Seguimiento al desempeño docente; por tal razón, se propone alinearlo a la norma ISO 9001:2015 para poder evaluar y mejorar el proceso de seguimiento al desempeño docente y garantizar la formación de los futuros profesionales del instituto.

Aunado a ello, el propósito de las organizaciones educativas se encuentra motivado a generar espacios de autorregulación. En el ámbito internacional se apuesta por la calidad educativa porque tiende a involucrar a todos los actores de la institución y depositan expectativas para la mejora de los procesos. A raíz de ello, los institutos de educación superior se regulan con las condiciones básicas de calidad para lograr la licenciatura que regenta la Superintendencia Nacional de Educación Superior Universitaria, y comprenden la necesidad de implementar Sistemas de Gestión de Calidad y en consecuencia, tener resultados óptimos para aplicar una acreditación con un modelo, en este caso fue con el modelo de Acreditación para Programas de Estudios de Institutos y Escuelas de Educación Superior del Sistema Nacional de Evaluación, Acreditación y Certificación. Ambos escenarios que tienen un carácter regulador tienen como base el cumplimiento de condiciones y estándares de calidad.

De acuerdo a la reunión del Dakar (2015), se planteó principales principios para la calidad educativa, indicando que una adecuada gestión para los procesos permite implementar bajo una normativa ISO una efectiva sistematización de la Gestión de la Calidad.

En la actualidad una educación de calidad es una necesidad mundial; pues como parte del Proyecto Educativo Nacional - PEN 2036 se indica que una educación de calidad combina criterios como la relación de la vida cotidiana con la vida en comunidad bajo la identificación de los valores como el respeto, valoración, paz y dialogo; y que la eficacia se logrará con el cumplimiento de los objetivos (PEN, 2020, p. 33).

Hoy en día, en el ámbito nacional se cuenta con el Modelo de Acreditación para Programas de Estudio de Institutos y escuelas de Educación Superior del Sistema Nacional de Evaluación, Acreditación y Certificación de la Calidad Educativa, cuyo modelo promulga el cumplimiento de 4 Dimensiones, 12 factores y 34 estándares cuyos parámetros buscan evidenciar el nivel de calidad de las organizaciones educativas, siendo fundamentales los estándares 
6 (Sistema de Gestión de Calidad); 13 (Selección, evaluación, capacitación y perfeccionamiento); 14 (plana docente adecuada) y 15 (Reconocimiento de las actividades de labor docente), para este estudio, pues, se busca evidenciar la relación significativa de los procesos del seguimiento del desempeño docente con respecto a los principios implementados de un sistema de gestión de calidad, ya que esta es una exigencia de regulación y es una herramienta que permite articular cada criterio de los estándares y demostrar que bajo el cumplimiento de los principios de calidad y el ciclo de mejora continua pueden forjar en la organización un nivel óptimo y satisfactorio de un proceso educativo.

Esta acción genera una inmediata mirada analítica a los procesos que gestiona el IESTP "Comunidad Teológica del Perú" y la revisión continua de un sistema de gestión de calidad existente, o la implementación inmediata de un sistema de gestión de calidad, ambos casos con una finalidad de generar espacios de autoevaluación que permitan regular las entradas de la organización educativa y propiciar un sólido, efectivo $y$ satisfactorio producto para la sociedad.

Con la implementación y cumplimiento de los requisitos y principios de un Sistema de Gestión de Calidad, ya que con ellos se pueden evidenciar el desarrollo de las personas que integran la organización educativa, y en este caso particular ejercer actividades para el óptimo seguimiento del desempeño docente, ya que es un componente necesario para comprender y mejorar los resultados de la formación académica.

El manuscrito analiza cómo la influencia de la implementación de un Sistema de Gestión de Calidad bajo la normativa ISO 9001:2015 en el seguimiento del desempeño docente en el IESTP "Comunidad Teológica del Perú".

\section{MÉTODO}

El trabajo realizado tuvo un alcance de tipo correlacional-causal porque se analizó la relación de los principios de un Sistema de Gestión de la Calidad basado en la norma ISO 9001:2015 y su influencia en los procesos del seguimiento del desempeño docente. La población fue de 60 personas, categorizado en tres grupos 12 administrativos, 18 docentes y 30 participantes entre estudiantes, auxiliares, padres de familia (otros). Fueron creados dos instrumentos, los cuales fueron validados por expertos, el primer cuestionario acerca se usó para medir la variable Gestión de la calidad, el cual estuvo constituido por 7 dimensiones relacionada a la implementación del SGC, descritos por 22 indicadores y a 05 escalas; y el segundo con referencia al Seguimiento al desempeño docente con 5 dimensiones, 30 indicadores y a 05 escalas, para obtener los datos se usó la técnica de la encuesta a la población declarada anteriormente.

Para obtener el grado de confiabilidad se realizó medianteelanálisis deconsistenciainternautilizando el método del Coeficiente Alfa de Cronbach que sirve para medir las correlaciones de las variables y evaluar la confiabilidad o la homogeneidad de las preguntas o ítems con alternativas policotómicas (Escala de Likert) (Hernández y Mendoza, 2018).

\section{RESULTADOS}

La Tabla 1 destaca los resultados de la encuesta acerca del sistema de Gestión de calidad implementado para garantizar un desarrollo confiable y dinámico de los procesos que se encuentran en su alcance, por tal razón, se presenta la evaluación de la implementación del SGC. 
Tabla 1. Variable Gestión de Calidad.

\begin{tabular}{llcccc}
\hline & & Frecuencia & Porcentaje & Porcentaje válido & Porcentaje acumulado \\
\hline \multirow{6}{*}{ Valores } & Nunca & 10 & 12,0 & 12,0 & 12,0 \\
& Casi Nunca & 11 & 16,0 & 16,0 & 28,0 \\
& A veces & 12 & 20,0 & 20,0 & 48,0 \\
& Casi Siempre & 12 & 20,0 & 20,0 & 68,0 \\
& Siempre & 15 & 32,0 & 32,0 & 100,0 \\
& Total & $\mathbf{6 0}$ & $\mathbf{1 0 0 , 0}$ & $\mathbf{1 0 0 , 0}$ & \\
\hline
\end{tabular}

Además, en la Tabla anterior, se evidenció que el IESTP "Comunidad Teológica del Perú", que un $32 \%$ de la población indicó que Siempre se analizó el Sistema de Gestión de Calidad, un 20\% de la población encuestada indicó que Casi Siempre o A Veces se analiza el SGC, un 16\% de la población indicó que Casi Nunca se analiza el SGC y un 12\% indicó que nunca se analiza el SGC.
Seguidamente se muestra la Tabla 2 donde se evidencia que un $40 \%$ de la población indicó que siempre se analiza el enfoque al cliente (estudiante), un $28 \%$ de la población que casi siempre se analiza el enfoque al cliente, un $24 \%$ indicó que a veces se analiza el enfoque al cliente y un $8 \%$ indicó que casi nunca se analiza el enfoque al cliente.

Tabla 2. Dimensión enfoque al cliente (estudiante).

\begin{tabular}{llcccc}
\hline & Frecuencia & Porcentaje & Porcentaje válido & Porcentaje acumulado \\
\hline \multirow{2}{*}{ Valores } & Casi Nunca & 10 & 8,0 & 8,0 & 8,0 \\
& A veces & 14 & 24,0 & 24,0 & 32,0 \\
& Casi Siempre & 16 & 28,0 & 28,0 & 60,0 \\
& Siempre & 20 & 40,0 & 40,0 & 100,0 \\
& Total & $\mathbf{6 0}$ & $\mathbf{1 0 0 , 0}$ & $\mathbf{1 0 0 , 0}$ & \\
\hline
\end{tabular}

En la Tabla 3, se muestra el análisis de la dimensión liderazgo en donde el $16 \%$ de la población encuestada indicó que siempre ha existido un liderazgo, el $24 \%$ indicó que casi siempre ha existido un liderazgo, un 16\% indicó que a veces ha existido un liderazgo, un $24 \%$ indicó que casi nunca ha existido un liderazgo y un 20\% manifestó que nunca ha existido un liderazgo.

Tabla 3. Dimensión Liderazgo.

\begin{tabular}{llcccc}
\hline & & Frecuencia & Porcentaje & Porcentaje válido & Porcentaje acumulado \\
\hline \multirow{2}{*}{ Valores } & Nunca & 12 & 20,0 & 20,0 & 20,0 \\
& Casi Nunca & 13 & 24,0 & 24,0 & 44,0 \\
& A veces & 11 & 16,0 & 16,0 & 60,0 \\
& Casi Siempre & 13 & 24,0 & 24,0 & 84,0 \\
& Siempre & 11 & 16,0 & 16,0 & 100,0 \\
& Total & $\mathbf{6 0}$ & $\mathbf{1 0 0 , 0}$ & $\mathbf{1 0 0 , 0}$ & \\
\hline
\end{tabular}


A continuación en la Tabla 4 con relación al compromiso del personal un $12 \%$ de la población consideró que no siempre se ha analizado el compromiso del personal, un $32 \%$ indicaba que casi siempre se analiza el compromiso del personal, un $16 \%$ de la población mencionaron que a veces se analiza el compromiso del personal, un $24 \%$ considera que casi nunca se analiza el compromiso del personal y un 16\% consideró que nunca se analiza el compromiso del personal.

Tabla 4. Compromiso del personal.

\begin{tabular}{clcccc}
\hline & & Frecuencia & Porcentaje & Porcentaje válido & Porcentaje acumulado \\
\hline \multirow{6}{*}{ Valores } & Nunca & 11 & 16,0 & 16,0 & 16,0 \\
& Casi Nunca & 13 & 24,0 & 24,0 & 40,0 \\
& A veces & 11 & 16,0 & 16,0 & 56,0 \\
& Casi Siempre & 15 & 32,0 & 32,0 & 88,0 \\
& Siempre & 10 & 12,0 & 12,0 & 100,0 \\
& Total & $\mathbf{6 0}$ & $\mathbf{1 0 0 , 0}$ & $\mathbf{1 0 0 , 0}$ & \\
\hline
\end{tabular}

En la siguiente Tabla 5, acerca del enfoque del proceso el 32\% de la población encuestada manifestó que siempre se analiza el enfoque a procesos, un $20 \%$ indicó que casi siempre se analiza el enfoque a procesos, un $20 \%$ indicó que a veces se analiza el enfoque a procesos, un $16 \%$ que casi nunca se analiza el enfoque a procesos, y un $12 \%$ que nunca se analiza el enfoque a procesos.

Tabla 5. Dimensión enfoque a procesos.

\begin{tabular}{llcccc}
\hline & Frecuencia & Porcentaje & Porcentaje válido & Porcentaje acumulado \\
\hline Valores & Nunca & 10 & 12,0 & 12,0 & 12,0 \\
& Casi Nunca & 11 & 16,0 & 16,0 & 28,0 \\
& A veces & 12 & 20,0 & 20,0 & 48,0 \\
& Casi Siempre & 12 & 20,0 & 20,0 & 68,0 \\
& Siempre & 15 & 32,0 & 32,0 & 100,0 \\
& Total & $\mathbf{6 0}$ & $\mathbf{1 0 0 , 0}$ & $\mathbf{1 0 0 , 0}$ & \\
\hline
\end{tabular}

Con relación a la dimensión mejora continua reflejada en la Tabla 6 , el $16 \%$ de la población encuestada indicó que siempre ha existido la mejora continua en la institución, un $24 \%$ dijo que casi siempre ha existido la mejora continua, un
16\% manifestó que a veces ha existido la mejora continua, un $24 \%$ indicó que casi nunca ha existido una mejora continua; y un $20 \%$ de la población encuestada concluyó que nunca ha existido la mejora continua.

Tabla 6. Dimensión mejora continua.

\begin{tabular}{clcccc}
\hline & & Frecuencia & Porcentaje & Porcentaje válido & Porcentaje acumulado \\
\hline \multirow{2}{*}{ Valores } & Nunca & 12 & 20,0 & 20,0 & 20,0 \\
& Casi Nunca & 13 & 24,0 & 24,0 & 44,0 \\
& A veces & 11 & 16,0 & 16,0 & 60,0 \\
& Casi Siempre & 13 & 24,0 & 24,0 & 84,0 \\
& Siempre & 11 & 16,0 & 16,0 & 100,0 \\
& Total & $\mathbf{6 0}$ & $\mathbf{1 0 0 , 0}$ & $\mathbf{1 0 0 , 0}$ & \\
\hline
\end{tabular}


En la dimensión toma de decisiones se encontró que el $12 \%$ de la población encuestada reflejó que siempre se ha analizado la toma de decisiones, un $32 \%$ indicó que casi siempre se ha analizado la toma de decisiones, un $16 \%$ que a veces se ha analizado la toma de decisiones, un $24 \%$ que casi nunca se ha analizado la toma de decisiones y un 16\% cerró que nunca se ha analizado la toma de decisiones. (Tabla 7).

Tabla 7. Dimensión toma de decisiones.

\begin{tabular}{clcccc}
\hline & & Frecuencia & Porcentaje & Porcentaje válido & Porcentaje acumulado \\
\hline \multirow{2}{*}{ Valores } & Nunca & 11 & 16,0 & 16,0 & 16,0 \\
& Casi Nunca & 13 & 24,0 & 24,0 & 40,0 \\
& A veces & 11 & 16,0 & 16,0 & 56,0 \\
& Casi Siempre & 15 & 32,0 & 32,0 & 88,0 \\
& Siempre & 10 & 12,0 & 12,0 & 100,0 \\
& Total & $\mathbf{6 0}$ & $\mathbf{1 0 0 , 0}$ & $\mathbf{1 0 0 , 0}$ & \\
\hline
\end{tabular}

Por otra parte, en la dimensión gestión de las relaciones se encontró que el $40 \%$ de la población encuestada indicó que siempre se analiza la gestión de las relaciones; un 28\% que casi siempre se analiza la gestión de las relaciones, un $24 \%$ que a veces se analiza la gestión de las relaciones y un $8 \%$ concluyó que casi nunca se analiza la gestión de las relaciones. (Tabla 8).

Tabla 8. Dimensión de la gestión de las relaciones

\begin{tabular}{llcccc}
\hline & Frecuencia & Porcentaje & Porcentaje válido & Porcentaje acumulado \\
\hline \multirow{2}{*}{ Valores } & Casi Nunca & 10 & 8,0 & 8,0 & 8,0 \\
& A veces & 14 & 24,0 & 24,0 & 32,0 \\
& Casi Siempre & 16 & 28,0 & 28,0 & 60,0 \\
& Siempre & 20 & 40,0 & 40,0 & 100,0 \\
& Total & 60 & 100,0 & 100,0 & $\mathbf{1 0 0 , 0}$ \\
\hline
\end{tabular}

La correlación que se obtuvo entre sistema de gestión de calidad y el seguimiento al desempeño docente de acuerdo con la prueba de inferencia, fue el coeficiente de correlación de 0.885 , por lo cual se infiere que existe una asociación interna positiva alta entre las variables estudiadas. No obstante, se obtuvo una sigma bilateral menor a 0.05 , por lo cual se rechaza la hipótesis nula, aceptándose la hipótesis alterna, infiriendo así que la Implementación de un SGC si influye en el Seguimiento al Desempeño Docente en el Instituto de Educación Superior Tecnológico Privado Comunidad Teológica del Perú 2020. (Tabla 9). 
Tabla 9. Correlación entre el Sistema de Gestión de Calidad y Seguimiento al Desempeño Docente.

\begin{tabular}{|c|c|c|c|c|}
\hline & & & $\begin{array}{c}\text { Sistema de Gestión } \\
\text { de Calidad }\end{array}$ & $\begin{array}{c}\text { Seguimiento al } \\
\text { Desempeño Docente }\end{array}$ \\
\hline \multirow[t]{6}{*}{ Rho de Spearman } & \multirow{3}{*}{$\begin{array}{l}\text { Sistema de } \\
\text { Gestión de } \\
\text { Calidad }\end{array}$} & Coeficiente de correlación & 1,000 &, $885^{\star \star}$ \\
\hline & & Sig. (bilateral) & . &, 000 \\
\hline & & $\mathrm{N}$ & 60 & 60 \\
\hline & \multirow{3}{*}{$\begin{array}{l}\text { Seguimiento } \\
\text { al Desempeño } \\
\text { Docente }\end{array}$} & Coeficiente de correlación &, $885^{\star *}$ & 1,000 \\
\hline & & Sig. (bilateral) &, 000 & . \\
\hline & & $\mathrm{N}$ & 60 & 60 \\
\hline
\end{tabular}

De acuerdo con la prueba de inferencia se obtuvo que el coeficiente de correlación entre el sistema de gestión de calidad y la dimensión didáctica fue de 0.998 , por lo cual se infiere que existe una asociación interna positiva alta entre las variables estudiadas. No obstante, se obtuvo un sigma bilateral menor a 0.05 , por lo cual se rechaza la hipótesis nula, aceptándose la hipótesis alterna, infiriendo así que la Implementación de un Sistema de Gestión de Calidad si influye en dimensión didáctica en el Instituto de Educación Superior Tecnológico Privado Comunidad Teológica del Perú 2020. (Tabla 10).

Tabla 10. Correlación entre el Sistema de Gestión de Calidad y Dimensión didáctica.

\begin{tabular}{lllcc}
\hline & & & $\begin{array}{c}\text { Sistema de Gestión } \\
\text { de Calidad }\end{array}$ & $\begin{array}{c}\text { Dimensión } \\
\text { Didáctica }\end{array}$ \\
\hline Rho de Spearman & $\begin{array}{l}\text { Sistema de Gestión } \\
\text { de Calidad }\end{array}$ & Coeficiente de correlación & 1,000 &, $988^{* *}$ \\
& & Sig. (bilateral) &. &, 000 \\
& Dimensión & Coeficiente de correlación & 60 & 60 \\
& Didáctica & Sig. (bilateral) &, $988^{* *}$ &, 000 \\
& & $\mathrm{~N}$ & 60 & 60 \\
\hline
\end{tabular}

Seguidamente se presenta la correlación entre el sistema de gestión de calidad y la dimensión personal, la cual manifestó que de acuerdo con la prueba de inferencia se obtuvo que el coeficiente de correlación fue de 0.944 , por lo cual se infiere que existe una asociación interna positiva alta entre las variables estudiadas. No obstante, se obtuvo un sigma bilateral menor a 0.05 , por lo cual se rechaza la hipótesis nula, aceptándose la hipótesis alterna, infiriendo así que la Implementación de un Sistema de Gestión de Calidad si influye en la dimensión personal en el Instituto de Educación Superior Tecnológico Privado Comunidad Teológica del Perú 2020. (Tabla 11). 
Tabla 11. Correlación entre el sistema de gestión de calidad y dimensión personal.

\begin{tabular}{lllcc}
\hline & & & $\begin{array}{c}\text { Sistema de Gestión } \\
\text { de Calidad }\end{array}$ & $\begin{array}{c}\text { Dimensión } \\
\text { Personal }\end{array}$ \\
\hline Rho de Spearman & $\begin{array}{l}\text { Sistema de Gestión } \\
\text { de Calidad }\end{array}$ & Coeficiente de correlación & 1,000 &, $944^{* *}$ \\
& & Sig. (bilateral) &. &, 000 \\
& Dimensión & Coeficiente de correlación & 60 & 60 \\
& Personal & Sig. (bilateral) &, $944^{* *}$ &, 000 \\
& & $\mathrm{~N}$ & 60 & 60 \\
\hline
\end{tabular}

La Tabla 12 destaca la correlación que arrojo el sistema de gestión de calidad con la dimensión institucional, en donde de acuerdo a la prueba de inferencia se obtuvo que el coeficiente de correlación es de 0.953 , por lo cual se infiere que existe una asociación interna positiva alta entre las variables estudiadas. No obstante, se obtuvo un sigma bilateral menor a 0.05 , por lo cual se rechaza la hipótesis nula, aceptándose la hipótesis alterna, infiriendo así que la Implementación de un Sistema de Gestión de Calidad influye en la dimensión Institucional en el Instituto de Educación Superior Tecnológico Privado Comunidad Teológica del Perú 2020.

Tabla 12. Correlación entre el Sistema de Gestión de Calidad y Dimensión Institucional.

\begin{tabular}{lllcc}
\hline & & & $\begin{array}{c}\text { Sistema de Gestión } \\
\text { de Calidad }\end{array}$ & $\begin{array}{c}\text { Dimensión } \\
\text { Institucional }\end{array}$ \\
\hline Rho de Spearman & $\begin{array}{l}\text { Sistema de Gestión } \\
\text { de Calidad }\end{array}$ & Coeficiente de correlación & 1,000 &, $953^{* *}$ \\
& & Sig. (bilateral) &. &, 000 \\
& Dimensión & Coeficiente de correlación & 60 & 60 \\
& Instituciona & Sig. (bilateral) &, $953^{* *}$ &, 000 \\
& & $\mathrm{~N}$ & 60 & 60 \\
\hline
\end{tabular}

De acuerdo con la prueba de inferencia en correspondencia entre correlación entre sistema sistema de gestión de calidad y la dimensión relaciones interpersonales se obtuvo que el coeficiente de correlación fue de 0.998 , por lo cual se infiere que existe una asociación interna positiva alta entre las variables estudiadas. No obstante, se obtuvo un sigma bilateral menor a 0.05 , por lo cual se rechaza la hipótesis nula, aceptándose la hipótesis alterna, infiriendo así que la Implementación de un Sistema de Gestión de Calidad si influye en la dimensión Relaciones interpersonales en el Instituto de Educación Superior Tecnológico Privado Comunidad Teológica del Perú 2020. (Tabla 13). 
Tabla 13. Correlación entre el Sistema de Gestión de Calidad y Dimensión Relaciones Interpersonales.

\begin{tabular}{|c|c|c|c|c|}
\hline & & & $\begin{array}{c}\text { Sistema de Gestión } \\
\text { de Calidad }\end{array}$ & $\begin{array}{l}\text { Dimensión Relaciones } \\
\text { Interpersonales }\end{array}$ \\
\hline \multirow[t]{6}{*}{ Rho de Spearman } & \multirow{3}{*}{$\begin{array}{l}\text { Sistema de Gestión } \\
\text { de Calidad }\end{array}$} & Coeficiente de correlación & 1,000 &, $988^{\star *}$ \\
\hline & & Sig. (bilateral) & . &, 000 \\
\hline & & $\mathrm{N}$ & 60 & 60 \\
\hline & \multirow{3}{*}{$\begin{array}{l}\text { Dimensión } \\
\text { Relaciones } \\
\text { Interpersonales }\end{array}$} & Coeficiente de correlación &, $988^{\star *}$ & 1,000 \\
\hline & & Sig. (bilateral) &, 000 & . \\
\hline & & $\mathrm{N}$ & 60 & 60 \\
\hline
\end{tabular}

De acuerdo con la prueba de inferencia se obtuvo que el coeficiente de correlación es de 0.944 , por lo cual se infiere que existe una asociación interna positiva alta entre las variables estudiadas. No obstante, se obtuvo un sigma bilateral menor a 0.05 , por lo cual se rechaza la hipótesis nula, aceptándose la hipótesis alterna, infiriendo así que la Implementación de un Sistema de Gestión de Calidad si influye en la dimensión Responsabilidad en el desempeño de sus funciones en el Instituto de Educación Superior Tecnológico Privado Comunidad Teológica del Perú 2020. (Tabla 14).

Tabla 14. Correlación entre el Sistema de Gestión de Calidad y Dimensión Responsabilidad.

\begin{tabular}{|c|c|c|c|c|}
\hline & & & $\begin{array}{c}\text { Sistema de Gestión } \\
\text { de Calidad }\end{array}$ & $\begin{array}{c}\text { Dimensión } \\
\text { Responsabilidad }\end{array}$ \\
\hline \multirow[t]{6}{*}{ Rho de Spearman } & \multirow{3}{*}{$\begin{array}{l}\text { Sistema de Gestión } \\
\text { de Calidad }\end{array}$} & Coeficiente de correlación & 1,000 & $944^{* *}$ \\
\hline & & Sig. (bilateral) & . & ,000 \\
\hline & & $\mathrm{N}$ & 60 & 60 \\
\hline & \multirow{3}{*}{$\begin{array}{l}\text { Dimensión } \\
\text { Responsabilidad }\end{array}$} & Coeficiente de correlación &, $944^{\star *}$ & 1,000 \\
\hline & & Sig. (bilateral) &, 000 & $\cdot$ \\
\hline & & $\mathrm{N}$ & 60 & 60 \\
\hline
\end{tabular}

Interpretación basada en el sistema de gestión de calidad y las dimensiones desarrolladas

a. De acuerdo a la prueba de inferencia se obtuvo que el coeficiente de correlación es de 0.885 , por lo cual se infiere que existe una correlación positiva alta entre las variables implementación de un SGC y el seguimiento al desempeño docente. Asimismo, se obtuvo una sigma bilateral menor a 0.05 , por lo que se infiere que la Implementación de un SGC sí influye en el Seguimiento al Desempeño Docente en el IESTP Comunidad Teológica del Perú. b. De acuerdo a la prueba de inferencia se obtuvo que el coeficiente de correlación es de 0.998 , por lo cual se infiere que existe una correlación positiva alta entre las variables implementación de un SGC y el seguimiento al desempeño docente. Asimismo, se obtuvo un sigma bilateral menor a 0.05 , por lo que se infiere que la Implementación de un SGC sí influye en la dimensión didáctica en el IESTP Comunidad Teológica del Perú.

c. De acuerdo a la prueba de inferencia se obtuvo que el coeficiente de correlación es de 0.944 , 
por lo cual se infiere que existe una correlación positiva alta entre las variables implementación de un SGC y el seguimiento al desempeño docente. Asimismo, se obtuvo un sigma bilateral menor a 0.05 , por lo que se infiere que la Implementación de un Sistema de Gestión de Calidad sí influye en la dimensión personal en el IESTP Comunidad Teológica del Perú 2020.

d. De acuerdo a la prueba de inferencia se obtuvo que el coeficiente de correlación es de 0.953 , por lo cual se infiere que existe una correlación positiva alta entre las variables implementación de un SGC y el seguimiento al desempeño docente. Asimismo, se obtuvo un sigma bilateral menor a 0.05 , por lo que se infiere que la Implementación de un SGC influye en la dimensión Institucional en el IESTP Comunidad Teológica del Perú.

e. De acuerdo a la prueba de inferencia se obtuvo que el coeficiente de correlación es de 0.998 , por lo cual se infiere que existe una correlación positiva alta entre las variables implementación de un SGC y el seguimiento al desempeño docente. Asimismo, se obtuvo un sigma bilateral menor a 0.05 , por lo que se infiere que la Implementación de un SGC sí influye en la dimensión Relaciones interpersonales en el IESTP Comunidad Teológica del Perú.

f. De acuerdo a la prueba de inferencia se obtuvo que el coeficiente de correlación es de 0.944 , por lo cual se infiere que existe una correlación positiva alta entre las variables implementación de un SGC y el seguimiento al desempeño docente. Asimismo, se obtuvo un sigma bilateral menor a 0.05 , por lo que se infiere que la Implementación de un SGC sí influye en la dimensión Responsabilidad en el desempeño de sus funciones en el IESTP Comunidad Teológica del Perú.

\section{Discusión}

En Carrera (2017), en su estudio realizado en Lima, acerca de un sistema de gestión de calidad basado en la norma ISO 9001:2015 para mejorar la eficacia de los procesos del Instituto Zegel IPAE; corroboró con datos cuantitativos, las evidencias y la mejora lograda con el desarrollo del sistema de gestión de calidad basado en la norma ISO 9001:2015; demostrando que sí se redujo o en algunos casos se erradicó los costos perdidos calculados con anterioridad, lo cual coincide con este estudio, el cual destaca que estos sistema contribuye a la mejora de la prestación de servicios educativos en el IESTP Comunidad Teológica del Perú.

Quispe (2017) en su estudio realizado en Huancayo elaboró un diseño que estuvo normado por los requisitos de la norma ISO 9001:2015, el cual estuvo dividido en 4 módulos relacionados 1 . La Comprensión de la organización y de su contexto, Comprensión de las necesidades y expectativas de las partes interesadas, Determinación del alcance del SGC, Política de la Calidad, Información Documentada, Matriz de enfoque de procesos, Mapa de procesos, Acciones para abordar los riesgos y oportunidades, Determinación de los requisitos para los servicios, Revisión de los requisitos para los productos y servicios, Enfoque, comunicación y satisfacción del cliente; 2. Objetivos de la Calidad y planificación para lograrlos, Planificación de los cambios, Procedimientos de comunicación interna, Procedimientos de sugerencias, quejas y reclamos; 3. Roles, responsabilidades y autoridades en la organización, Competencia e inducción del personal, Conocimiento de la organización, Inducción de personal, recursos de seguimiento y medición, infraestructura y ambiente de trabajo, finalmente el módulo 4. Planificación y control operacional, control de los procesos, productos $\mathrm{y}$ servicios suministrados externamente, tipo $\mathrm{y}$ 
alcance del control de proveedores, Información para los proveedores externos, Seguimiento, Análisis y Medición Auditoría interna, Revisión por la dirección y Mejora. Como resultado de la implementación del Sistema de Gestión de Calidad ISO 9001:2015 se logró mejorar la satisfacción de los clientes con $74 \%$ de satisfacción y se logró alcanzar el nivel 4 en el cuestionario y reducir las quejas y reclamos hasta el nivel 5; al igual que en este estudio se hace necesario siempre la implementación de este sistemas porque arroja resultados positivos en la gestión, tal como se destaca en los resultados arrojados en este estudio.

El estudio de Castillo (2018) concluyó que deben integrarse metodologías activas, practicando valores y dando cumplimiento a la normativa de la entidad, coincidiendo pero también ampliando estos valores con el buen desempeño docente basado en la correlación del Sistema de Gestión de Calidad y su seguimiento al desempeño, el cual se extendió en los trabajadores y los estudiantes los cuales influyen positivamente como la columna vertebral en la prestación de un buen sistema de calidad en la institución educativa.

Seguidamente Espinoza (2019) en su estudio sobre el seguimiento a docentes universitarios en las estrategias metodológicas fundamentadas en el constructivismo de la carrera de Pedagogía, realizado en la Universidad Católica de Santiago de Guayaquil - Ecuador; concluyó que era necesario crear un modelo de seguimiento al docente para poder afianzar y proponer estrategias metodológicas fundamentadas en el constructivismo, en este estudio se pudo cuantificar el seguimiento a la labor docene a fin de garantizar la mejoro en la calidad educativa necesaria en el sistema de gestión de calidad se estudia para implementar en el IESTP Comunidad Teológica del Perú.

\section{CONCLUSIONES}

En el PEI del IESTP "Comunidad Teológica del Perú” 2018 - 2020 busca fortalecer la gestión institucional y para ello, establece que se debe implementar un Sistema de Gestión de Calidad, debido que no contaban con un mecanismo que gestione los procesos de la enseñanza y aprendizaje. El estudio basado en el análisis realizado sobre la calidad de los servicios evaluados pudo determinar la necesidad de continuar usando un sistema de gestión de calidad.

En este sentido los docentes tienen poco conocimiento de los procedimientos del seguimiento a su desempeño docente y desconocen de los resultados obtenidos en las evaluaciones que el IESTP realiza internamente, a raíz de ello, se analiza los resultados obtenidos cuyos resultados indican que la implementación de un SGC se encuentra en un nivel bueno con un $88.0 \%$ estos resultados manifiestan que la institución viene brindando una buena atención a los administrados y que estas acciones están alineadas a los objetivos organizacionales de calidad del instituto, estos resultados son corroborados por el nivel de desempeño de los trabajadores que se encuentran en un $100 \%$, debido que ellos manifiestan que la implementación del SGC les ha permitido fomentar una cultura organizacional con un trasfondo de trabajo en equipo, por lo que fortalecen su desempeño.

\section{REFERENCIAS}

Carrera Salazar, G. M. (2017). Propuesta de implementación de un sistema de gestión de calidad basado en la norma ISO 9001: 2015 para mejorar la eficacia de los procesos del Instituto Zegel IPAE 
Castillo Quispe, T. J. (2018). Mejorar el desempeño docente en uso de materiales educativos en sesiones de aprendizaje de estudiantes de la I.E.P. $\mathrm{N}^{\circ}$ Chocorosi - JDCH Azángaro -Puno

CNA-Chile. (2006). Acreditación. [Consulta el 13 de enero de 2021] disponible en: https://www. cnachile.cl/Paginas/Inicio.aspx

CONEAU-Argentina. (1995). Acreditación de Carreras de Grado. [Consultado el 13 de enero de 2021] disponible en https://www.coneau.gob. ar/coneau/acreditacion-de-carreras/carrerasde-grado/

Consejo Nacional de Educación. (2020). El proyecto Educativo Nacional al 2036. Lima: Ministerio de Educación y Consejo Nacional de Educación. Recuperado de https://www.cne. gob.pe/uploads/publicaciones/2020/proyectoeducativo-nacional-al-2036.pdf

De la Garza Aguilar, J. (s.f.). Consejo Para la Acreditación de La Educación Superior, A.C. (COPAES), MÉXICO. Contribución especial II.11. Acreditación de la educación superior en América Latina y el Caribe https://upcommons.upc.edu/bitstream/ handle/2099/7540/18_295-298.pdf

Espinoza Robles, G. (2019). Elseguimientoa docentes universitarios en las estrategias metodológicas fundamentales en el constructivismo de la carrera de Pedagogía de la Universidad Católica de Santiago de Guayaquil
Hernández-Sampieri, R. \& Mendoza, C (2018). Metodología de la investigación. Las rutas cuantitativa, cualitativa y mixta, Ciudad de México, México: Editorial Mc Graw Hill Education, Año de edición: 2018, ISBN: 978-14562-6096-5, $714 \mathrm{p}$

International Organization for Standardization, 2015. ISO 9001:2015: Sistemas de gestión de la calidad - Requisitos. Ginebra: ISO

James, Evans y William, Lindsay. (2005). Administración y control de calidad. Thomson Editores, Madrid. España

Quispe Luya, K. G. (2017). Sistema de gestión de calidad para la mejora del servicio de los talleres y laboratorios para la enseñanza - aprendizaje de la Universidad Continental.

SENUDO, (2020). Proyecto normativo del reglamento delicenciamiento para universidades nuevas. Disponible en: https://www.sunedu.gob. pe/reglamento-procedimiento-licenciamientouniversidades-nuevas/

SINEACE. (2016). Modelo de Acreditación para programas de Estudios de Institutos y Escuelas de Educación Superior. Lima, Perú 